\title{
2q37 Deletion syndrome confirmed by high-resolution cytogenetic analysis
}

\author{
Eun-Kyung Cho, MD, \\ Jinsup Kim, MD, \\ Aram Yang, MD, \\ Sung Yoon Cho, MD, PhD, \\ Dong-Kyu Jin, MD, PhD
}

Departments of Pediatrics, Samsung Medical Center, Sungkyunkwan University School of Medicine, Seoul, Korea
Received: 27 September, 2016

Revised: 18 October, 2016

Accepted: 26 October, 2016

Address for correspondence:

Sung Yoon Cho, MD, PhD

Department of Pediatrics, Samsung Medical Center, Sungkyunkwan University School of Medicine, 81 Irwonro, Gangnam-gu, Seoul 06351, Korea

Tel: +82-2-3410-3539

Fax: +82-2-3410-0043

E-mail: nadri1217@naver.com

https://orcid.org/0000-0003-2913059X
Chromosome 2q37 deletion syndrome is a rare chromosomal disorder characterized by mild to moderate developmental delay, brachydactyly of the third to fifth digits or toes, short stature, obesity, hypotonia, a characteristic facial appearance, and autism spectrum disorder. Here, we report on a patient with 2 q37 deletion presenting with dilated cardiomyopathy (DCMP). Congenital heart malformations have been noted in up to $20 \%$ of patients with 2 q37 deletions. However, DCMP has not been reported in 2q37 deletion patients previously. The patient exhibited the characteristic facial appearance (a flat nasal bridge, deep-set eyes, arched eyebrows, and a thin upper lip), developmental delay, mild mental retardation, peripheral nerve palsy, and Albright hereditary osteodystrophy (AHO)-like phenotypes (short stature and brachydactyly). Conventional chromosomal analysis results were normal; however, microarray-based comparative genomic hybridization revealed terminal deletion at $2 \mathrm{q} 37.1 \mathrm{q} 37.3$. In addition, the patient was confirmed to have partial growth hormone (GH) deficiency and had shown a significant increase in growth rate after substitutive GH therapy. Chromosome 2 q37 deletion syndrome should be considered in the differential diagnosis of patients presenting with AHO features, especially in the presence of facial dysmorphism. When patients are suspected of having a $2 q 37$ deletion, high-resolution cytogenetic analysis is recommended.

Keywords: Chromosome 2q37 deletion syndrome, Albright's hereditary osteodystrophy, Brachydactyly, Dilated cardiomyopathy, Array based comparative genomic hybridization

\section{Introduction}

Deletions of the distal 2q37 region involve the last cytogenetic band on the long arm of chromosome 2, ${ }^{1)}$ and the clinical features of terminal microdeletions of $2 \mathrm{q} 37$ were first described in 1989 by Gorski et al. ${ }^{2}$. The incidence is unclear, but more than 115 patients carrying isolated, primarily terminal deletions with breakpoints at or within chromosome $2 \mathrm{q} 37$ have been reported ${ }^{1)}$. This disease shows facial dysmorphism, including a prominent forehead; sparse, arched eyebrows; deep-set eyes; midface hypoplasia; a depressed nasal bridge; a thin upper lip; and various pinna anomalies, ranging from fleshy and/or anteverted lobules to microtia ${ }^{3}$. In addition, brachymetacarpia and brachymetaphalangia, typical signs of Albright hereditary osteodystrophy (AHO), are well described in $2 \mathrm{q} 37$ microdeletion syndrome ${ }^{4)}$. In contrast to pseudohypoparathyroidism Ia or pseudopseudohypoparathyroidism, patients with 2q37 deletion syndrome do not have any mutations in GNAS, and they lack renal parathyroid hormone resistance and soft-tissue ossification ${ }^{5)}$.

About $30 \%$ of patients with 2q37 deletions show major malformations, including congenital heart disease, gastrointestinal or genitourinary anomalies, and central nervous system malformations ${ }^{3)}$. Affected individuals often have mild to moderate developmental delay, 
mental retardation, and hypotonia ${ }^{6}$. Autistic features also occur in association with terminal deletions with breakpoints at any subband of $2 \mathrm{q} 37^{7)}$

Here, we report a patient with 2q37 deletion confirmed by high-resolution cytogenetic analysis (i.e., microarray-based comparative genomic hybridization [array-CGH]). The patient presented with dilated cardiomyopathy (DCMP), short stature, and AHO-like phenotypes as well as characteristic facial features. DCMP associated with $2 \mathrm{q} 37$ deletion syndrome has not been reported in the literature.

\section{Case report}

The patient was the first child of healthy nonconsanguineous Korean parents. She was born by cesarean section at 35 weeks of gestation with a birth weight of 2,400 $\mathrm{g}$ (25th-50th percentile). She showed left microtia and cupping of the right pinna. Conventional chromosome analysis revealed 46, XX. Her younger sister was healthy, and her family history was unremarkable. Showing an asymmetric facial expression, she was diagnosed with right peripheral-type facial nerve palsy at the age of 2 years and the symptoms persisted. She had mild developmental delay. She walked with support at 15 months of age and started speaking single words at 12 months of age. The patient now attends normal school, although she has moderate learning difficulties.

At the age of 7 years, she was admitted to the hospital due to coughing and vomiting. The chest radiograph showed cardiomegaly, and the echocardiogram revealed myocardial dysfunction with a dilated left ventricle. She was diagnosed as having myocarditis with DCMP and heart failure. After three weeks, she recovered with medical treatment. Four months later, she was admitted to the hospital again due to fever, dizziness, and chest discomfort. She was referred to Samsung Medical

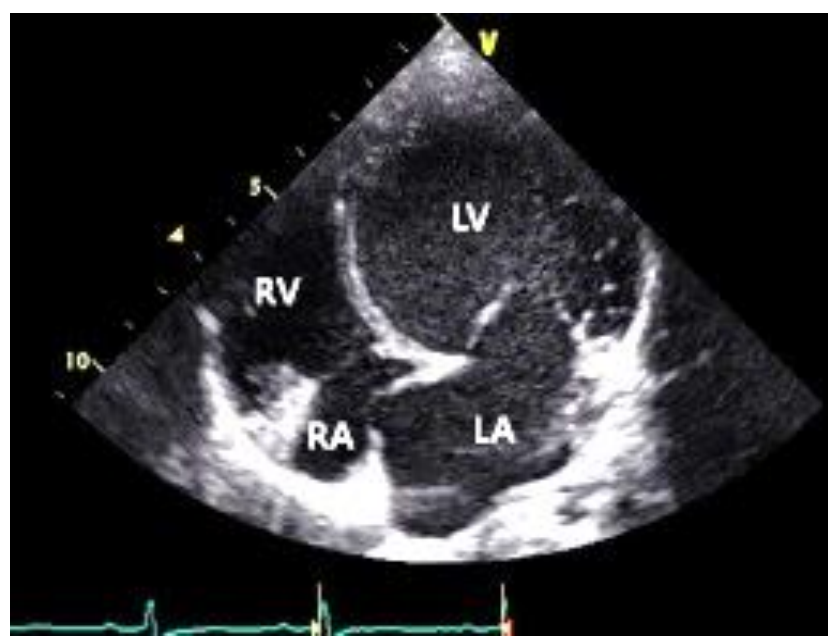

Fig. 1. Two-dimensional echocardiography at diagnosis showing dilated cardiomyopathy. Note the extremely dilated left ventricle. The left ventricular end-diastolic dimension was $5.9 \mathrm{~cm}$, and the ejection fraction was $26 \%$. LV, left ventricle ; $R V$, right ventricle ; $L A$, left atrium ; $R A$, right atrium.
Center because of her DCMP with congestive heart failure. The echocardiogram revealed severe myocardial dysfunction with a dilated left ventricle (Fig. 1). The estimated left ventricular ejection fraction (EF) was $26 \%$, and the left ventricular enddiastolic dimension was $5.9 \mathrm{~cm}$. Heart magnetic resonance imaging (MRI) revealed DCMP without myocardial fibrosis, and there was no evidence of congenital heart defect or coronary artery anomaly. In addition, a heart biopsy revealed nonspecific findings. The exact cause of the patient's DCMP was unknown. On the 10th day, a follow-up echocardiogram showed improved cardiac function and decreased heart size, and she was discharged with medications (captopril, furosemide, spironolactone, carvedilol, and digoxin).

At the age of 10 years, she visited the outpatient clinic of pediatric endocrinology due to short stature. Given her height of $124.3 \mathrm{~cm}(-2.25$ standard deviation [SD]) and her weight of $24.5 \mathrm{~kg}(-1.88 \mathrm{SD})$, her body mass index was $15.9 \mathrm{~kg} / \mathrm{m}^{2}$ $(-0.26 \mathrm{SD})$. Her midparental height was $163.5 \mathrm{~cm}$. A physical examination revealed that she was in Tanner stage I for breast development. She exhibited a flat nasal bridge, deep-set eyes, arched eyebrows, and a thin upper lip as well as abnormalities of both ears. Temporal bone computed tomography showed bony atresia of the left external auditory canal, while her hearing evaluation result was normal. In addition, she presented bilateral

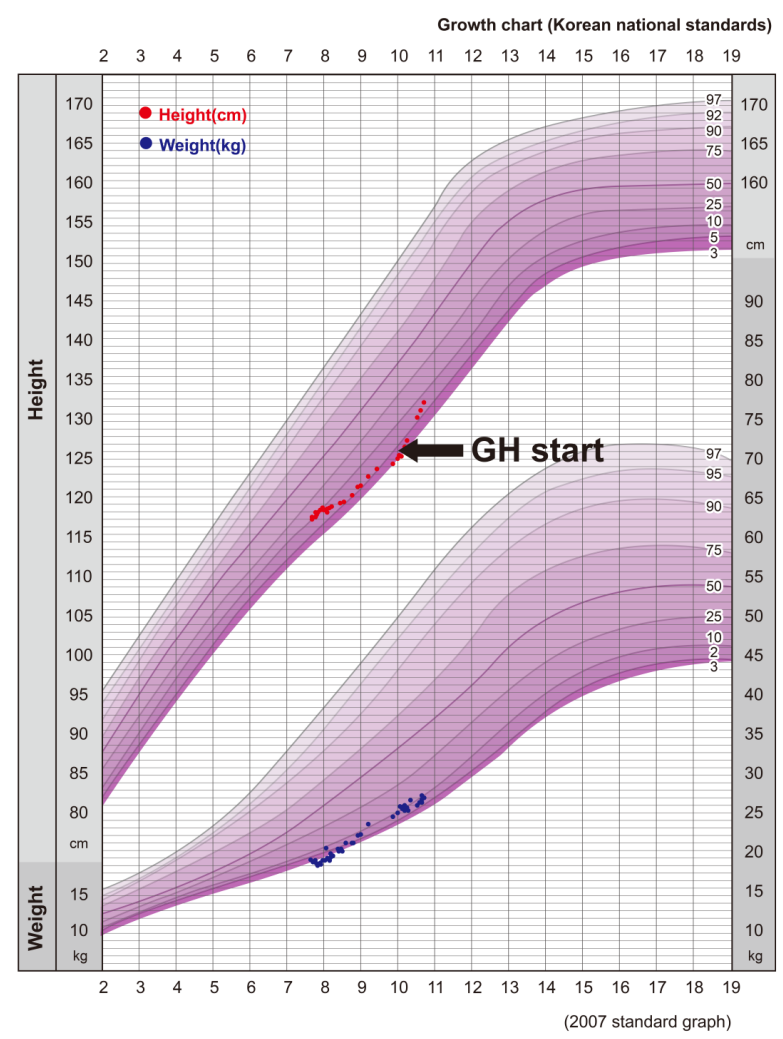

Fig. 2. Patient's growth chart. In the figure, height measurements from 7 years to 10.8 years are reported. Growth hormone (GH) therapy was started at 10 years of age (arrow) and led to a significant growth velocity increase, as shown in the figure. 
brachymetatarsia of the third to fifth toes and brachymetacarpia of the third to fifth metacarpal bones (Fig. 2). Ophthalmologic examinations showed congenital myogenic ptosis, and she underwent bilateral levator resection surgery at the age of 10 years. She often showed waddling gait and hyperflexibility of the hips. The patient did not have autistic features.

The data from biological tests (i.e., routine blood cell count, calcium, phosphate, creatinine, serum alkaline phosphatase, 25-hydroxyvitamin D, thyroid hormone, parathyroid hormone, and urine analysis) appeared within the normal ranges. The serum insulin-like growth factor I (IGF-I) value was lower than the age-specific reference ranges $(64.9 \mathrm{ng} / \mathrm{mL} ; z$-score, -2.4). Radiographs of her hands showed a bone age of 10 years at a chronological age of 10 years and 3 months. The renal ultrasonography results were normal.

Cytogenetic analysis was performed again using peripheral blood lymphocytes with the 550-band level, including GTG banding, to evaluate her short stature and dysmorphic features. Genomic DNA was isolated from the peripheral blood leukocytes using the Wizard Genomic DNA Purification kit (Promega, Madison, WI, USA). An Affymetrix Cytogenetics Whole-Genome 2.7M Array (Affymetrix Inc., Santa Clara, CA, USA) was used for copy number variation (CNV) analysis according to the manufacturer's instructions. Copy number data were analyzed using the Chromosome Analysis Suite 1.1 (Affymetrix Inc.). The array contains approximately 400,000 single nucleotide polymorphism and 2,370,000 unique nonpolymorphic probes for determination of the CNV. Segments with $>50$ markers and $>100 \mathrm{~kb}$ were considered to be CNVs. The hg19 assembly was used as a reference. It revealed the deletion of 2 q37.1 to 2qter. Finally, array-CGH revealed terminal deletion (about $8.3 \mathrm{Mb}$ ) at 2q37.1q37.3 (Fig. 3). The array-CGH results of both parents were normal.

In the growth hormone (GH) stimulation test, the peak level of GH was $7.56 \mathrm{ng} / \mathrm{ml}$ by L-dopa and $8.63 \mathrm{ng} / \mathrm{mL}$ by glucagon. Sella MRI revealed a pars intermedia cyst. Human GH therapy $(0.55 \mathrm{mg} / \mathrm{kg} / \mathrm{wk})$ was started when she was 10 years old, and her growth velocity has improved (Fig. 4). After eight months of GH therapy, her height was $133.1 \mathrm{~cm}(-0.9$ SD) with a significant increase in growth rate to $8.8 \mathrm{~cm}$. In addition, IGF-1 value increased to $266.6 \mathrm{ng} / \mathrm{mL}$ after eight months of GH therapy.
The patient did not have any heart failure symptoms while on medications, and her latest echocardiogram at the age of 10 years showed improved cardiac function (an EF of 53.6\% and left ventricular end-diastolic dimension of $46.8 \mathrm{~mm}$ ).

\section{Discussion}

We report a Korean patient with deletion of $2 q 37$. In the case of $2 q$ subtelomeric deletions, significant variability in clinical presentation is apparent, but almost all patients have some degree of mental retardation and facial dysmorphism ${ }^{6}$. Our patient had the following features similar to the reported phenotype of 2q37 deletion: characteristic facial features, ear abnormalities, ptosis, brachydactyly, mild mental retardation, developmental delay, and short stature. Another congenital defect found in our patient was peripheral nerve palsy, but it is nonspecific for the $2 \mathrm{q} 37$ deletion phenotype.

Unusually, our patient suffered from DCMP without any congenital structural defects. Congenital heart malformations have been noted in up to $20 \%$ of patients with 2 q 37 deletions ${ }^{3)}$.

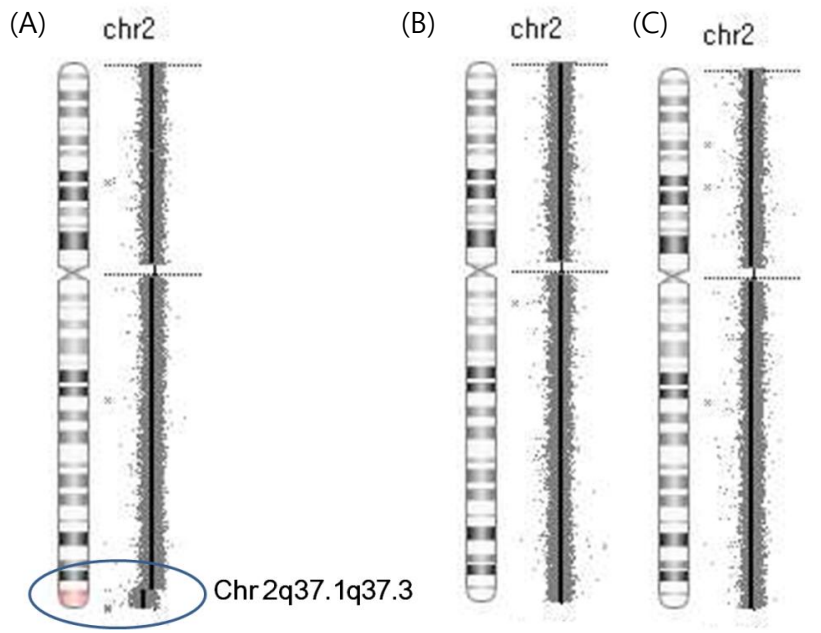

Fig. 4. Results of array-comparative genomic hybridization. (A) The patient showed loss of 2q37.1q37.3. The patient's father (B) and mother (C) showed normal chromosomes.

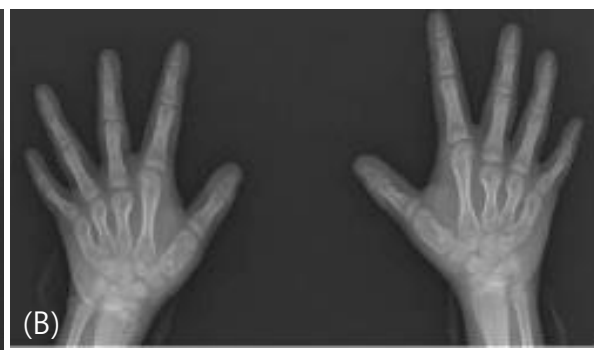

Fig. 3. Plain radiographs of feet (A) and hands (B) at 7 years of age, showing shortening of the third, fourth, and fifth metatarsals and metacarpals. 
Septal defects are most common, and aortic coarctation has been described ${ }^{3)}$. However, DCMP has not been reported in $2 \mathrm{q} 37$ deletion patients, and the relationship between DCMP and $2 \mathrm{q} 37$ deletion is not clear.

Our patient presented with short stature and was diagnosed as having partial GH deficiency. To date, there have been only 3 reports of GH deficiency in patients with $2 \mathrm{q} 37$ deletions $^{6,8,9)}$. Two cases were confirmed as $2 \mathrm{q} 37$ deletions ${ }^{6,9)}$, and 1 case was confirmed as a distal $2 \mathrm{p}$ duplication and $2 \mathrm{q}$ deletion with severe short stature and pituitary hypoplasia ${ }^{8)}$. All 3 reports noted a significant increase in growth rate after substitutive GH therapy, as shown in our patient.

Chromosome analysis confirms the diagnosis of $2 \mathrm{q} 37$ microdeletion syndrome in $80 \%-85 \%$ of affected individuals ${ }^{10)}$. The largest telomeric deletion is about $10 \mathrm{Mb}$, while the smallest is around 3 to $4 \mathrm{Mb}$ in the $2 \mathrm{q} 37$ chromosomal region. Deletion size does not appear to correlate well with phenotype ${ }^{11)}$. The size of the $2 \mathrm{q} 37$ deletion was $8.3 \mathrm{Mb}$ in our patient. The genes involved with $2 \mathrm{q} 37.1 \mathrm{q} 37.3$ deletion are UTG1A4, UGT1A1, MLPH, COL6A3, PER2, TWIST2, HDAC4, NDUFA10, AGXT, KIF1A, PASK, and D2HGDH. The phenotypic implications of most of them remain unknown. Among the involved genes with a known phenotype, HDAC4 has notably been established as being responsible for brachymetaphalangia and intellectual disability ${ }^{12)}$. In about $15 \%-20 \%$ of cases, the conventional karyotype is normal because of the small size of the deleted region ${ }^{10}$. Our patient showed a normal karyotype in the conventional chromosomal analysis; however, 2 q37 microdeletion was identified by high-resolution cytogenetic analysis (i.e., array-CGH). Therefore, when patients are suspected of having a 2q37 deletion, high-resolution cytogenetic analysis is recommended ${ }^{3}$. Fluorescence in situ hybridization or array-CGH can be considered to confirm the diagnosis ${ }^{3)}$.

It is important that chromosome $2 \mathrm{q} 37$ microdeletion be considered when patients have AHO features, especially in the presence of characteristic facial dysmorphism. We report a case of 2q37 deletion with DCMP, peripheral nerve palsy, and partial GH deficiency that responded well to GH therapy. When patients are suspected of having a 2q37 deletion, high-resolution cytogenetic analysis is recommended.

\section{Conflict of interest}

No potential conflict of interest relevant to this article was reported.

\section{Acknowledgments}

This study was supported by a grant from Samsung Medical Center (grant number: GFO2160061).

\section{References}

1. Leroy C, Landais E, Briault S, David A, Tassy O, Gruchy
$\mathrm{N}$, et al. The 2q37-deletion syndrome: an update of the clinical spectrum including overweight, brachydactyly and behavioural features in 14 new patients. Eur J Hum Genet 2013;21:602-12.

2. Gorski JL, Cox BA, Kyine M, Uhlmann W, Glover TW. Terminal deletion of the long arm of chromosome 2 in a mildly dysmorphic hypotonic infant with karyotype 46,XY,del(2)(q37). Am J Med Genet 1989;32:350-2.

3. Falk RE, Casas KA. Chromosome $2 q 37$ deletion: clinical and molecular aspects. Am J Med Genet C Semin Med Genet 2007;145C:357-71.

4. Simşek-Kiper PO, Utine GE, Alanay Y, Aktaş D, Alikaşifoğlu $\mathrm{M}$, Boduroğlu K. A rare case of $2 \mathrm{q} 37$ microdeletion with Albright hereditary osteodystrophy-like phenotype. Turk J Pediatr 2011;53:558-60.

5. Mehraein Y, Pfob M, Steinlein O, Aichinger E, Eggert M, Bubendorff V, et al. 2q37.3 Deletion syndrome: two cases with highly distinctive facial phenotype, discordant association with schizophrenic psychosis, and shared deletion breakpoint region on 2q37.3. Cytogenet Genome Res 2015;146:33-8.

6. Kitsiou-Tzeli S, Sismani C, Ioannides M, Bashiardes S, Ketoni A, Touliatou V, et al. Array-CGH analysis and clinical description of $2 \mathrm{q} 37.3$ de novo subtelomeric deletion. Eur J Med Genet 2007;50:73-8.

7. Casas KA, Mononen TK, Mikail CN, Hassed SJ, Li S, Mulvihill JJ, et al. Chromosome 2q terminal deletion: report of 6 new patients and review of phenotypebreakpoint correlations in 66 individuals. Am J Med Genet A 2004;130A:331-9.

8. Vetro A, Pagani S, Silengo M, Severino M, Bozzola E, Meazza C, et al. Severe growth hormone deficiency and pituitary malformation in a patient with chromosome 2 p25 duplication and 2q37 deletion. Mol Cytogenet 2014;7:41.

9. Wilson LC, Leverton K, Oude Luttikhuis ME, Oley CA, Flint J, Wolstenholme J, et al. Brachydactyly and mental retardation: an Albright hereditary osteodystrophy-like syndrome localized to 2q37. Am J Hum Genet 1995;56:4007.

10. Doherty ES, Lacbawan FL. 2q37 Microdeletion syndrome. In: Pagon RA, Adam MP, Ardinger HH, Wallace SE, Amemiya A, Bean LJH, et al., editors. GeneReviews(R). Seattle (WA): University of Washington, Seattle; 1993-2017.

11. Aldred MA, Sanford RO, Thomas NS, Barrow MA, Wilson LC, Brueton LA, et al. Molecular analysis of 20 patients with 2q37.3 monosomy: definition of minimum deletion intervals for key phenotypes. J Med Genet 2004;41:433-9.

12. Williams SR, Aldred MA, Der Kaloustian VM, Halal F, Gowans G, McLeod DR, et al. Haploinsufficiency of HDAC4 causes brachydactyly mental retardation syndrome, with brachydactyly type E, developmental delays, and behavioral problems. Am J Hum Genet 2010;87:21928. 